\title{
Oxygen Overlayers on Pd(111) Studied by Density Functional Theory ${ }^{\dagger}$
}

\author{
Mira Todorova, Karsten Reuter, and Matthias Scheffler* \\ Fritz-Haber-Institut der Max-Planck-Gesellschaft, Faradayweg 4-6, D-14195 Berlin, Germany
}

Received: January 29, 2004; In Final Form: April 19, 2004

\begin{abstract}
By use of density-functional theory we analyze the on-surface adsorption of oxygen on $\operatorname{Pd}(111)$ for coverages up to 1 monolayer and compare the results with corresponding data for the other late $4 \mathrm{~d}$ transition metals, namely, $\mathrm{Ru}, \mathrm{Rh}$, and $\mathrm{Ag}$. Besides the known effect of the continued $d$-band filling on the oxygen-metal bond strength, we also discern trends in the adsorption geometries, work functions, and electron density of states. The repulsive lateral interactions in the overlayer give rise to a pronounced reduction of the adsorption energy at higher on-surface coverages. In fact, for oxygen coverages $\theta>0.5$ monolayers, the thermodynamic equilibrium phase of $\mathrm{O} / \mathrm{Pd}(111)$ is known to be a surface oxide. The calculations reported in this paper show that on-surface adlayers at such higher coverages, that may exist as metastable phases, still possess qualitatively the same surface chemical bond as that which is found at low coverages. The dependence of the surface relaxation on oxygen coverage exhibits some unexpected behavior.
\end{abstract}

\section{Introduction}

Oxygen chemisorbed on single-crystal metal surfaces constitutes an important model system for surface science, and it represents an initial step toward the formation of metal oxides (see, for example, refs 1-4 and references therein). Furthermore, the late $4 \mathrm{~d}$ transition metals (and the oxygen-metal interaction) play a noticeable role for oxidation catalysis. ${ }^{5}$ In particular, for the close-packed $\mathrm{Pd}(111)$ surface numerous experimental studies have been performed, e.g., refs 6-13. The picture arising from this work is that for temperatures $T>200 \mathrm{~K}$ oxygen adsorption is dissociative and for a coverage of $\theta=0.25$ monolayer (ML) a $(2 \times 2)$ ordered overlayer is formed, where the oxygen adatoms occupy the 3-fold face-centered cubic (fcc) hollow sites. At higher coverages, on-surface adsorption seems to compete with the formation of a (surface) oxide, and depending on the preparation conditions, a $(\sqrt{ } 3 \times \sqrt{ } 3) \mathrm{R} 30^{\circ},{ }^{6}$ a $(1 \times 1),{ }^{7,11}$ and a so-called "complex"6,13 structure have been observed by lowenergy electron diffraction (LEED). In a combined experimental and density functional theory (DFT) study, the atomic structure behind the "complex" LEED pattern was recently identified as a surface oxide containing about $0.7 \mathrm{ML}$ of oxygen. ${ }^{13}$

In a recent trend study (from $\mathrm{Ru}$ to $\mathrm{Ag}$ ), we addressed the thermodynamics of the transition from the oxygen overlayer to subsurface incorporation and (surface) oxide formation. ${ }^{3}$ The main motivation of the present work is to achieve a deeper insight into details of the oxygen-metal chemical bond. For this purpose, we focus on the on-surface chemisorption of $\mathrm{O} / \mathrm{Pd}$ (111) in the coverage range between $\theta=0.25$ and $1 \mathrm{ML}$, analyzing the binding energies, as well as surface geometries, work functions, and electron densities. We use DFT together with the all-electron full-potential linearized augmented plane wave (FP-LAPW) method and will also compare the present results to those of corresponding DFT studies concerned with $\mathrm{O}$ adsorption on $\mathrm{Ru}(0001),{ }^{14} \mathrm{Rh}(111),{ }^{15}$ and $\mathrm{Ag}(111) .{ }^{16}$

The next section gives a brief summary of the technical details of our DFT calculations. Then, section III describes the various

\footnotetext{
† Part of the special issue "Gerhard Ertl Festschrift".

* To whom correspondence should be addressed. E-mail: scheffler@ fhi-berlin.mpg.de.
}

results: (III-A) for Pd bulk and the clean Pd(111) surfaces, (IIIB.1) for the $\mathrm{O} / \mathrm{Pd}(111)$ binding energies, (III-B.2) for the adsorbate-induced surface relaxations, and (III-B.3) for the surface electronic structure. In section IV, we conclude and give an outlook for future studies.

\section{Theory}

The DFT calculations were performed with the all-electron FP-LAPW method ${ }^{17-19}$ using the generalized gradient approximation $(\mathrm{GGA})^{20}$ for the exchange-correlation functional. All surfaces were modeled by supercells, employing a symmetric slab consisting of seven $\operatorname{Pd}(111)$ layers. A vacuum region of $17 \AA$ ensures the decoupling of consecutive slabs. Oxygen was adsorbed at both sides of the slab, and all atomic positions within the oxygen adlayer and the two outermost Pd layers were fully relaxed. The total energy of the $\mathrm{Pd}$ atom required for the determination of the bulk cohesive energy was obtained by calculating a spin-polarized, nonsymmetry averaged $\mathrm{Pd}$ atom in a huge supercell of dimensions (13 $\AA$ × $14 \AA \times 15 \AA$ ).

The FP-LAPW basis set parameters are as follows: $R_{\mathrm{MT}}^{\mathrm{Pd}}=$ $2.25 \mathrm{bohr}, R_{\mathrm{MT}}^{\mathrm{O}}=1.3 \mathrm{bohr}$, wave function expansion inside the muffin tin spheres up to $l_{\max }^{\mathrm{wf}}=12$, and potential expansion up to $l_{\max }^{\mathrm{pot}}=4$. The energy cutoff for the plane wave representation in the interstitial region between the muffin tin spheres was $E_{\mathrm{wf}}^{\mathrm{max}}=17$ Ry for the wave functions and $E_{\mathrm{pot}}^{\mathrm{max}}=169 \mathrm{Ry}$ for the potential. The $\mathrm{Pd} 4 \mathrm{~s}$ and $4 \mathrm{p}$ and the $\mathrm{O} 2 \mathrm{~s}$ orbitals were treated as "semi-core states" by adding corresponding local orbitals to the LAPW basis set. A $(12 \times 12 \times 1)$ Monkhorst-Pack (MP) grid was used for the $(1 \times 1)$ Brillouin zone integration. For the larger surface cells, the grid is reduced accordingly to keep the same sampling of the reciprocal space, and $\Gamma$-point sampling was used for the calculation of the isolated $\mathrm{Pd}$ atom.

Below we will discuss the average binding energy which is defined as

$$
E_{\mathrm{b}}(\theta)=-\frac{1}{N_{\mathrm{O}}}\left(E_{\mathrm{O} / \mathrm{Pd}(111)}-E_{\mathrm{Pd}(111)}-\frac{N_{\mathrm{O}}}{2} E_{\mathrm{O}_{2}}\right)
$$


where $N_{\mathrm{O}}$ is the total number of $\mathrm{O}$ atoms present in the unit cell at the considered coverage $\theta . E_{\mathrm{O} / \mathrm{Pd}(111)}, E_{\mathrm{Pd}(111)}$, and $E_{\mathrm{O}_{2}}$ are the total energies of the slab containing the oxygen adsorbate, the corresponding clean Pd slab, and the total energy of an isolated oxygen molecule, respectively. The definition is such that a positive $E_{\mathrm{b}}$ value indicates that the dissociative adsorption of $\mathrm{O}_{2}$ is exothermic at $T=0 \mathrm{~K}$.

To keep the required interstitial plane-wave cutoff as low as possible, the FP-LAPW calculations were done with as large as possible, nonoverlapping muffin tin spheres. Because of the small bond length of the oxygen molecule, its total energy, $E_{\mathrm{O}_{2}}$, cannot be calculated directly with the muffin tin radius, $R_{\mathrm{MT}}^{\mathrm{O}}=$ $1.3 \mathrm{bohr}$, chosen for the surface calculations. We therefore used a procedure explained in refs 15 and 21, which exploits the relation $E_{\mathrm{O}_{2}}=2 E_{\mathrm{O}}^{\mathrm{atom}}-D$. Here, $E_{\mathrm{O}}^{\mathrm{atom}}$ is the total energy of an isolated oxygen atom and $D$ is the theoretical $\mathrm{O}_{2}$ binding energy. It is well known that DFT results for the oxygen atom and the $\mathrm{O}_{2}$ molecule are subject to noticeable GGA-induced errors. We note, however, that this will neither affect the discussion below nor the conclusions, because we focus on differences between binding energies at different coverages, $\Delta E_{\mathrm{b}}$.

The numerical accuracy of our results is primarily limited by the FP-LAPW basis set and the finite thickness of the slab. To estimate the importance of the various numerical approximations, several test calculations have been performed. For example, we increased the energy cutoff for the plane-wave basis set from 17 to $24 \mathrm{Ry}$. While the computed binding energies decreased by about $0.2 \mathrm{eV} /$ atom, the change in $\Delta E_{\mathrm{b}}$ was less than $20 \mathrm{meV}$. A similarly small change was obtained when a denser $(15 \times 15 \times 1)$ MP mesh was used. To determine the error due to the finite slab size, we tested 9 and 11 layer thick slabs. Although the absolute binding energies were lowered by up to $65 \mathrm{meV}$, the $\Delta E_{\mathrm{b}}$ values were within $\pm 30 \mathrm{meV}$. Considering all the test calculations, we estimate the numerical uncertainty for the binding energy differences to be $\pm 50 \mathrm{meV}$. As it will become apparent below, this does not affect our discussion and conclusions.

\section{Results}

A. Bulk Pd and Clean Pd(111). The DFT-GGA lattice constant for Pd is calculated as $a=3.94 \AA$ (neglecting zeropoint vibrations). The bulk modulus is $B=163 \mathrm{GPa}$, as obtained using the Murnaghan equation of state. The experimental values at room temperature (obviously including vibrational contributions) are $a_{\exp }=3.89 \AA$ and $B_{\exp }=181 \mathrm{GPa} .{ }^{22}$ The slight overestimation of the lattice constant (about $+2 \%$ ) and the (corresponding) underestimation of $B$ are in line with analogous studies for other late $4 \mathrm{~d}$ TMs. ${ }^{14-16}$ Previous pseudopotential calculations for Pd gave $a=3.98 \AA^{23}$ and $a=4.01 \AA,^{12}$ overestimating the experimental value even more. Our calculated cohesive energy is $E_{\mathrm{coh}}=3.64 \mathrm{eV} /$ atom, which compares well with the experimental result, $E_{\text {coh,exp }}=3.94 \mathrm{eV}^{22}$

For the clean $\operatorname{Pd}(111)$ surface, we find that the top interlayer spacing, $d_{12}$, is slightly contracted $(-0.1 \%)$ with respect to the bulk value, which is $d_{\mathrm{b}}=2.28 \AA$. The second Pd interlayer spacing, $d_{23}$, exhibits a similarly insignificant expansion by $+0.1 \%$. These results are in good agreement with the analyses of available LEED data. In refs 24-26, only a slight expansion of $d_{12}$ is reported (about $+1 \%$ ), which, according to the authors, might also be due to the presence of some residual hydrogen at the surface. In refs 12,27 , and 28 , no indication of surface relaxations was found.

With its almost nonexistent surface relaxation, clean $\mathrm{Pd}(111)$ differs from the two elements to its left in the periodic table,

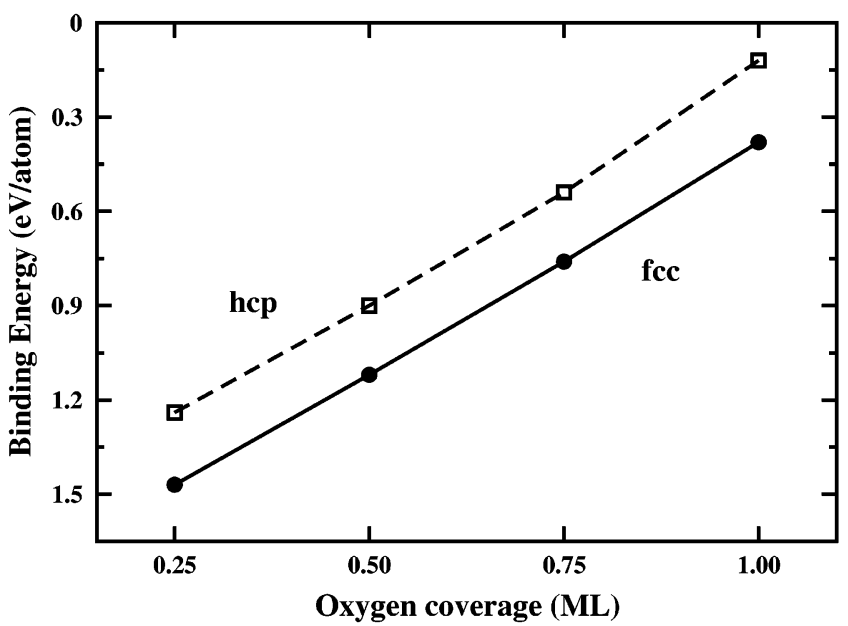

Figure 1. Calculated binding energies of oxygen on $\operatorname{Pd}(111)$ in fcc (-) and hcp ( $\square$ ) hollow sites. The energy zero is $1 / 2$ the energy of a free $\mathrm{O}_{2}$ molecule. The lines are guides to the eye.

for which appreciable first interlayer contractions of $-3.9 \%$ at $\mathrm{Ru}(0001)^{29}$ and $-1.8 \%$ at $\operatorname{Rh}(111)^{15}$ were computed. This behavior reflects the variation in the nature of the bonds with d-band occupation as discussed, for example, by Methfessel, Henning, and Scheffler. ${ }^{30}$ Briefly, the bonding goes from mainly $\mathrm{d}-\mathrm{d}$ attraction and repulsive sp pressure (in $\mathrm{Ru}$ ) to largely $\mathrm{s}$ bonding and $\mathrm{d}-\mathrm{d}$ repulsion (in $\mathrm{Ag}$ ). Thus, the inward relaxation is largest for a half-full $\mathrm{d}$ band $(\mathrm{Ru})$, because the spill out of $\mathrm{s}$ electron density into the vacuum reduces the s pressure at the surface, and the cut $\mathrm{d}$ bonds of the top Ru layer enable a stronger $\mathrm{d}$ bonding with the second layer. For the noble metal Ag, where the s electrons actuate the bonding, their spill out at the surface into the vacuum leads to a bond weakening and thus to a small expansion of $d_{12} \cdot{ }^{30}$

B. Oxygen Overlayers on Pd(111). 1. Energies. To investigate the on-surface chemisorption of oxygen on $\operatorname{Pd}(111)$ over the sub-monolayer range, the coverage was increased from 0 to $1 \mathrm{ML}$ in quarter monolayer steps. As expected, ${ }^{8,23}$ we find oxygen adsorption to be most favorable in the 3-fold hollow sites. The calculated binding energies are displayed in Figure 1 , which reveals a preference for the fcc over the hexagonal close-packed (hcp) hollow site by about $0.2 \mathrm{eV}$. This value is almost the same for the whole coverage range considered. The energy of oxygen in bridge sites is about $0.5 \mathrm{eV}$ less favorable than in the fcc hollow sites. This holds for $1 / 4 \mathrm{ML}$ as well as for $1 \mathrm{ML}$ coverage $((2 \times 2)$ and $(1 \times 1)$ structures, respectively), which suggests that also the diffusion energy barrier does not vary much with coverage.

The binding energy difference between the two hollow sites (cf. Figure 1) is similar to that found for $\mathrm{O} / \mathrm{Rh}(111),{ }^{15}$ while for $\mathrm{O} / \mathrm{Ru}(0001),{ }^{14}$ it is larger for lower coverages and only reduces to $0.1 \mathrm{eV}$ for $1 \mathrm{ML}$. The found preference for the fcc hollow sites on fcc $\operatorname{Pd}(111)$ is consistent with results for the other late TMs: On the basal planes, oxygen is always found to occupy those sites that continue the bulk layer sequence (i.e., fcc on fcc $\operatorname{Rh}(111)$ and $\operatorname{Ag}(111)$, hcp on hcp $\operatorname{Ru}(0001)) .{ }^{14-16}$ In this respect, and similar to the conclusions of the study by Seitsonen et al., ${ }^{12}$ our calculations cast doubt on the interpretation of ion scattering data by Steltenpohl and Memmel, determining the hcp site as the most stable $\mathrm{O}$ adsorption site on $\mathrm{Pd}(111) .{ }^{31}$ However, we note that a more complex surface modification, as for example a parallel displacement of the $\mathrm{O} / \mathrm{Pd}$ double layer at the surface (e.g., at steps), giving rise to an hcp 


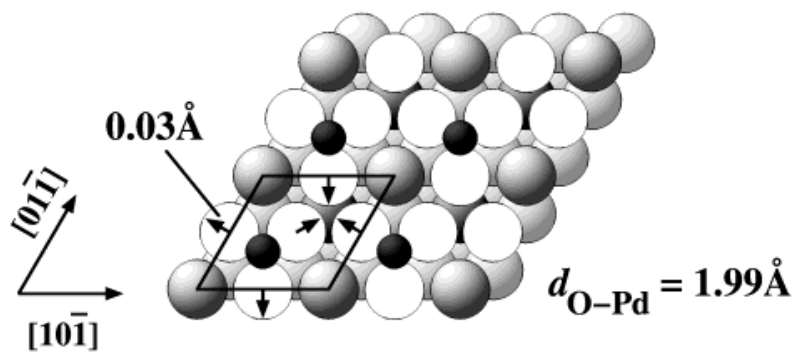

[111]

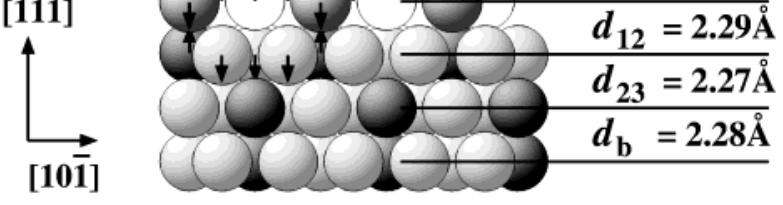

Figure 2. Top and side views of the atomic geometry of the $\mathrm{O}(2 \times$ 2)/Pd(111) structure. Small, dark gray circles represent $O$ atoms; large, white and gray circles represent Pd atoms; those lying in the same plane and are equivalent under the 3 -fold symmetry have the same color. The arrows indicate the direction of the displacements of the substrate atoms with respect to the ideal bulk positions.

stacking of the top three Pd layers, cannot be ruled out at this point (see an analogous discussion for the $\mathrm{O} / \mathrm{Ru}(0001)$ system in ref 21).

The marked decrease in the binding energy with increasing coverage (cf. Figure 1) reflects the overall repulsive lateral interactions in the overlayer. In general, this favors the formation of dilute adlayer structures over dense islands. However, on the basis of the few ordered structures calculated here, we cannot draw conclusions as to which ordered or disordered overlayers may actually form as a thermodynamic phase (allowing also for metastable states). Such a topic requires the evaluation of the partition function, e.g., via constructing a lattice gas Hamiltonian, for which a far more extensive database and a subsequent Monte Carlo study are necessary. ${ }^{32}$

The computed binding energy decrease over the submonolayer coverage range on $\operatorname{Pd}(111)$ is found to agree even in magnitude with that computed for oxygen on $\mathrm{Ru}(0001)$, Rh(111), and $\operatorname{Ag}(111) .^{3}$ The binding energy of $\mathrm{O}$ on $\operatorname{Pd}(111)$, however, is significantly weaker than that of $\mathrm{O}$ on $\mathrm{Ru}(0001)$ and $\mathrm{Rh}(111)$ but stronger than that of $\mathrm{O}$ on $\operatorname{Ag}(111) .^{3}$ This progression of the binding energy throughout the sequence of late $4 \mathrm{~d}$ TMs is a consequence of the continued filling of the $\mathrm{d}$ band toward the right of the periodic table, yielding an increased occupation of antibonding oxygen-metal states that weaken the $\mathrm{O}-$ metal bond (cf. section III.B.3). In contrast to the highly exothermic binding energy of oxygen on $\mathrm{Ru}(0001),{ }^{14}$ the bond strength on $\operatorname{Ag}(111)$ is already so low that dissociative adsorption would be endothermic for coverages beyond 0.5 ML. ${ }^{16}$ $\mathrm{O} / \mathrm{Pd}(111)$ is a borderline case where the full ML coverage is only just exothermic at $T=0 \mathrm{~K}$ in our DFT-GGA calculations. We recall, however, that for coverages $\theta>0.7 \mathrm{ML}$, the thermodynamic equilibrium structure of $\mathrm{O} / \mathrm{Pd}(111)$ is a surface oxide. ${ }^{13}$ Nevertheless, it is possible that higher-coverage onsurface overlayers exist as metastable structures, in particular at low temperatures.

2. Surface Relaxations. The calculated surface geometries of the $\theta=0.25,0.5$, and $0.75 \mathrm{ML}$ overlayers are shown in Figures 2,3 , and 4 . From these systems only the $\mathrm{O}(2 \times 2)$ adlayer has been identified experimentally, and our geometry parameters are in very good agreement with a LEED intensity analysis, ${ }^{12}$ as summarized in Table 1. Oxygen pushes its three Pd neighbors radially away $(0.03 \AA)$ and lifts them up. This is counteracted

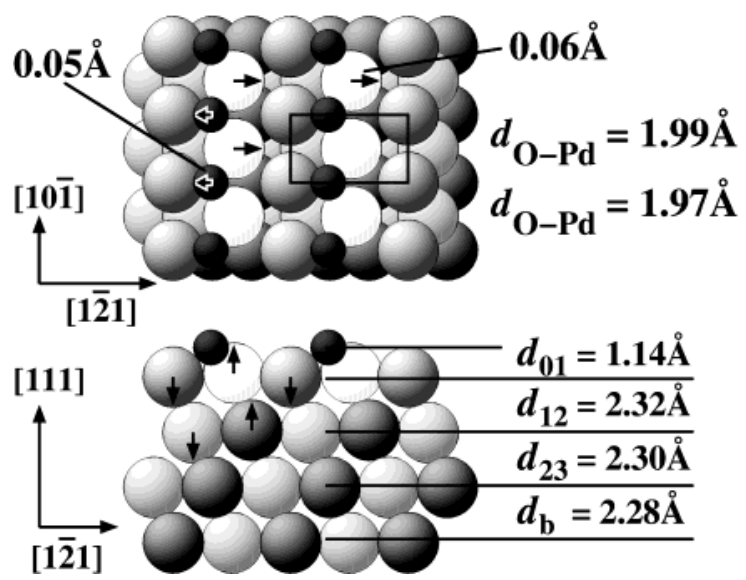

Figure 3. Top and side views of the atomic geometry of the $\mathrm{O}(2 \times$ 1)/Pd(111) structure. Small, dark gray circles represent $O$ atoms; large, white and gray circles represent $\mathrm{Pd}$ atoms; those lying in the same plane and are equivalent under the 3-fold symmetry have the same color. The arrows indicate the direction of the displacements of the substrate atoms with respect to the ideal bulk positions.

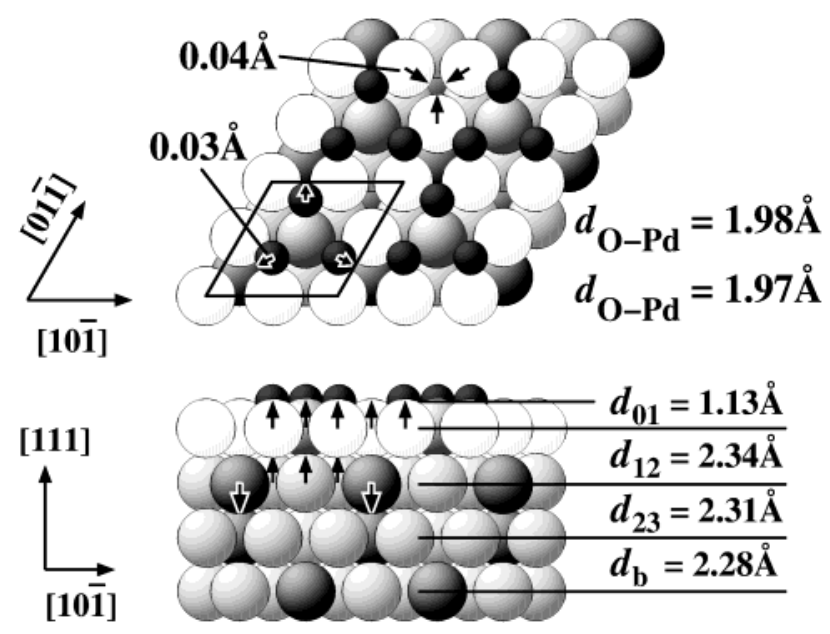

Figure 4. Top and side views of the atomic geometry of the $3 \mathrm{O}(2 \times$ 2)/Pd(111) structure. Small, dark gray circles represent $O$ atoms; large, white and gray circles represent Pd atoms; those lying in the same plane and are equivalent under the 3-fold symmetry have the same color. The arrows indicate the direction of the displacements of the substrate atoms with respect to the ideal bulk positions.

TABLE 1: Calculated Geometry Parameters (in Å) for the $\mathbf{O}(2 \times 2) / \mathbf{P d}(111)$ Structure with Oxygen in fcc Hollow Sites ${ }^{a}$

\begin{tabular}{lllc}
\hline $\begin{array}{c}\text { geometry } \\
\text { parameters }\end{array}$ & \multicolumn{1}{c}{ DFT-GGA } & \multicolumn{1}{c}{ LEED } & PP-DFT-GGA \\
\hline$d_{\text {O-Pd }}$ & 1.99 & & 1.97 \\
$d_{01}$ & 1.16 & $1.17 \pm 0.03$ & 1.16 \\
$d_{12}$ & $2.29(+0.4 \%)$ & $2.30 \pm 0.03(+2.0 \%)$ & 2.34 \\
$d_{23}$ & $2.27(-0.5 \%)$ & & \\
$d_{\mathrm{b}}$ & 2.28 & 2.25 & 2.31 \\
$\Delta z_{1}$ & 0.09 & $0.07 \pm 0.03$ & 0.07 \\
$\Delta z_{2}$ & 0.10 & $0.08 \pm 0.05$ &
\end{tabular}

${ }^{a}$ For the interlayer distances $d$, the center of mass of each Pd layer is used and the percent change with respect to the bulk distance, $d_{b}$, is noted as well. $d_{\mathrm{O}-\mathrm{Pd}}$ is the $\mathrm{O}-\mathrm{Pd}$ bond length, and $\Delta z_{1}$ and $\Delta z_{2}$ are the buckling in the first and second outermost layers, respectively. The experimental LEED results and the pseudopotential (PP-DFT-GGA) values are from ref 7.

by a downward movement of the non-O-coordinated Pd atom, leading to a modest first and second layer buckling of 0.09 and $0.10 \AA$, respectively. Overall this changes the slight compression of the first interlayer spacing of the clean surface, $d_{12}$, into a 


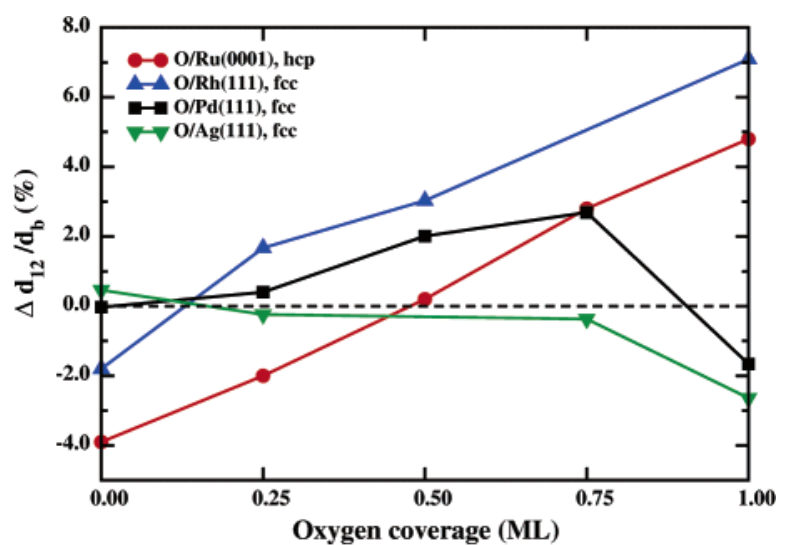

Figure 5. Coverage dependence of the interlayer spacing of the top metal layers, $d_{12}$, relative to that of the bulk interlayer spacing, $d_{\mathrm{b}}$. For buckled layers the center of gravity is used. The results for $\mathrm{Rh}(111)$ are from ref 15 and those for $\mathrm{Ru}(0001)$ and $\mathrm{Ag}(111)$ from ref 29

small expansion, but in total, the top interlayer Pd relaxation is still negligible.

For the $\mathrm{O}(2 \times 1)$ structure $(\theta=0.5 \mathrm{ML})$, the calculated relaxation pattern is slightly more complex (cf. Figure 3 ). There is an upward-sideward displacement of the 2-fold O-coordinated surface $\mathrm{Pd}$ atoms, and related to that, the $\mathrm{O}$ adatoms do not sit any more exactly in the center of the fcc hollow site. Furthermore, there is a downward shift of the 1-fold Ocoordinated Pd surface atoms, yielding a substantial 0.16- $\AA$ firstlayer buckling. The oxygen-induced increase of $d_{12}$ found for the $\theta=0.25 \mathrm{ML}$ phase continues and results in a $+2.0 \%$ expansion with respect to the bulk interlayer distance. Also, $d_{23}$ is expanded ( $\left.+0.9 \%\right)$, and the second layer is found to be buckled by $0.10 \AA$.

Going to higher coverage $(\theta=0.75 \mathrm{ML})$, i.e., to the $3 \mathrm{O}(2 \times$ 2) structure, the buckling is less pronounced than for $\theta=0.5$ ML. We now find a buckling of $0.09 \AA$ for the first Pd layer and of $0.05 \AA$ for the second one (cf. Figure 4). The overall relaxation pattern remains very much the same as before: The oxygen adatoms are slightly pushed away from the highest coordinated Pd atoms, and the first and second interlayer spacings are further expanded (now by $+2.6 \%$ and $+1.3 \%$ ).

It therefore comes as a surprise that this layer relaxation trend is not continued, and it is even reversed, for the $\theta=1 \mathrm{ML}$ overlayer. Now we find that the $d_{12}$ distance is reduced (compared to the bulk interlayer spacing) by $-1.7 \%$ (for $\theta=$ $0.75 \mathrm{ML}$, we had found a $+2.6 \%$ expansion). Concerning $d_{23}$, we obtain an expansion of $+1.8 \%$, somewhat in line with the results for the lower coverage overlayers. The violation of the trend of $d_{12}(\theta)$ for $0<\theta \leq 0.75 \mathrm{ML}$ is distinct to the behavior found for $\mathrm{Ru}$ and $\mathrm{Rh}$. Some similarity exists, however, to Ag. Figure 5 summarizes all these results.

Detailed structural data for oxygen adsorption in the submonolayer range on $\mathrm{Ru}(0001), \mathrm{Rh}(111)$, and $\mathrm{Ag}(111)$ are available from DFT calculations, ${ }^{14-16}$ where the study by Ganduglia-Pirovano and Scheffler on $\mathrm{Rh}(111)^{15}$ employed a similar FP-LAPW setup as the present study. The O/Ru and the $\mathrm{O} / \mathrm{Ag}$ systems were calculated using the pseudopotential approach. To exclude uncertainties in the comparison, possibly caused by the pseudopotential approximation, we recalculated the four considered oxygen overlayers with our FP-LAPW setup also for $\mathrm{Ru}(0001)$ and $\mathrm{Ag}(111) .{ }^{29}$ Overall, we obtained very good agreement with the surface geometries described in the pseudopotential studies. Where slight differences were found, they could be traced back to the corresponding potential-energy surface minimum being very shallow. This is particularly the case for the more noble metals and leads then to some uncertainty when employing conventional structural relaxation procedures. After ensuring a proper location of the minimum, we intriguingly found the local relaxation pattern around the $\mathrm{O}$ atoms in each of the four afore described overlayer structures (lateral displacements, buckling) to be highly similar for all four metals. What changes are the calculated changes in $d_{12}$ for the different metals, and Figure 5 displays them as a function of coverage, with oxygen atoms always sitting in the most stable adsorption sites. For the clean surfaces, we see the abovementioned strong contraction for $\mathrm{Ru}(0001)$ that is gradually reduced when going to $\mathrm{Rh}(111), \mathrm{Pd}(111)$, and $\mathrm{Ag}(111)$. On $\mathrm{Ru}$ (0001) and $\mathrm{Rh}(111)$, oxygen adsorption induces a strong increase of the outermost interlayer distance, that raises almost linearly with coverage. This behavior reflects the strong oxygen - metal bonds formed in these systems that weaken the remaining back bonds of the first-layer metal atoms to the underlying substrate. At $\operatorname{Ag}(111)$, on the other hand, $d_{12}$ stays at first more or less constant, and then at full ML coverage it even contracts. This has been attributed to the rather weak $\mathrm{O}-\mathrm{Ag}$ bonds that are no longer able to significantly disturb the metal surface geometry. ${ }^{16}$ Within this picture, $\mathrm{O} / \mathrm{Pd}(111)$ appears again as a borderline case. Initially, the $\mathrm{O}-\mathrm{Pd}$ bond is strong enough to induce a progressive expansion of the first interlayer distance with $\mathrm{O}$ coverage, cf. Figure 5. Yet, compared to Ru and Rh, the bond strength is weak and the expansion less pronounced, and in the $\mathrm{O}(1 \times 1)$ structure (possibly because the repulsive lateral interactions among the adsorbates have further weakened the $\mathrm{O}-\mathrm{Pd}$ bond) the outward relaxation can no longer be sustained and the surface prefers to contract like Ag. Last but not least, we like to recall that for all these systems, with the exception of $\mathrm{Ru}$, surface oxide formation is expected before the full monolayer is formed. ${ }^{3}$ Correspondingly, the above discussion may be somewhat of academic relevance only, namely, to gain an understanding of trends in energies and atomic relaxations. Nevertheless, it is possible that some of these structures can indeed be prepared experimentally as metastable phases.

3. Electronic Structure. Let us now consider the oxygeninduced change in the work function, $\Delta \Phi(\theta)$, as a function of oxygen coverage. For the clean surface, which is taken as the reference, the work function is calculated as $5.25 \mathrm{eV}$. The experimental value for $\mathrm{Pd}(111)$ is $5.6 \mathrm{eV} .{ }^{33}$ Thus, the difference between theory and experiment is noticeable $(0.35 \mathrm{eV})$, which is along the line with other GGA calculations. The local-density approximation is apparently giving a better description of the work function and a value in close agreement with the measured one. ${ }^{30}$ The oxygen induced work function change is however more similar for both functionals, and Figure 6 shows that $\Delta \Phi$ $(\theta)$ rises almost linearly with coverage for both the fcc and the hcp hollow adsorption sites. The increase is slightly larger for the fcc site, for which we get: $\Delta \Phi(\theta=1 \mathrm{ML})=1.54 \mathrm{eV}$. Both curves exhibit the typical curvature reflecting a depolarization with increasing coverage, but this effect is apparently rather modest for the $\mathrm{O} / \mathrm{Pd}(111)$ system. Also shown in Figure 6 is the adsorbate-induced dipole moment, and here the depolarization is well visible: The adsorbate-induced dipole moment decreases by about $20 \%$ when going from $\theta=0.25$ ML to $\theta=1 \mathrm{ML}$.

This adsorbate dipole moment is a direct consequence of the adsorbate-induced change of the electron density, which is shown in Figure 7 for the $\mathrm{O}(2 \times 2)$ structure with $\mathrm{O}$ in fcc sites. To be precise, Figure 7 displays what is typically called the "difference electron density". ${ }^{34}$ This quantity is obtained by subtracting from the electron density of the $\mathrm{O} / \mathrm{Pd}(111)$ system 


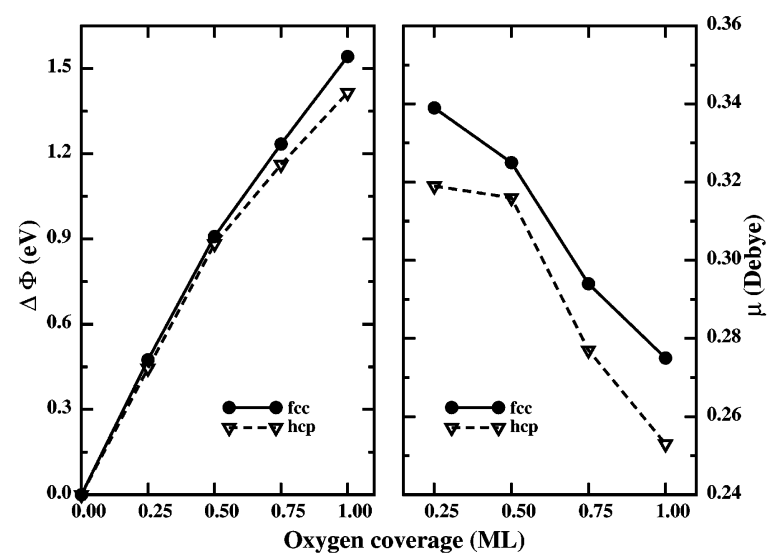

Figure 6. Calculated work function change (left) and dipole moment (right) for $\mathrm{O}$ on $\mathrm{Pd}(111)$ as a function of the oxygen coverage. Shown are results for oxygen atoms in the two most stable on-surface adsorption sites, i.e., fcc $(\bullet)$ and hcp $(\nabla)$ hollow sites.

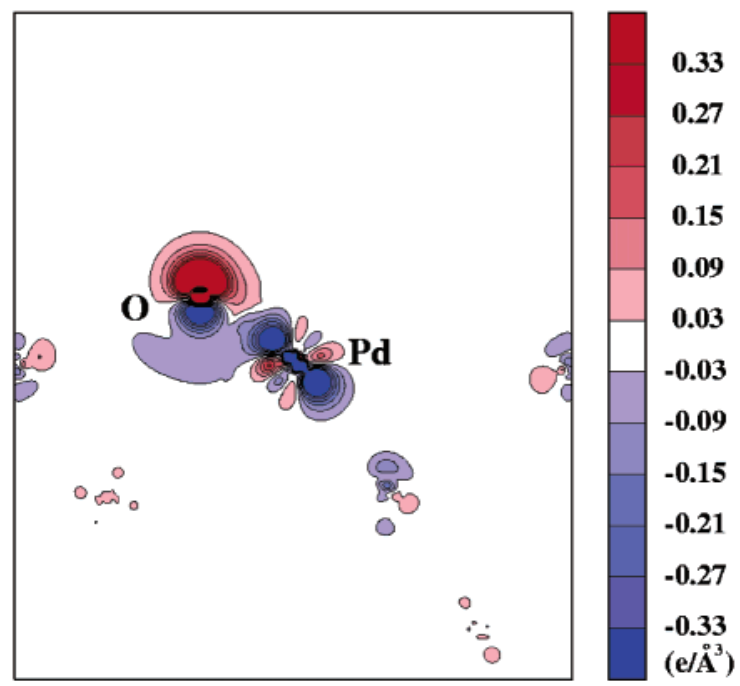

Figure 7. Difference electron density plot for $1 / 4 \mathrm{ML} \mathrm{O}$ adsorbed on $\operatorname{Pd}(111)$ in fcc hollow sites. The contour plot depicts the (112) plane perpendicular to the (111) surface passing through the center of the oxygen atoms. Areas of electron accumulation (red) and depletion (blue) have positive and negative signs, respectively; contour lines are drawn at $0.06 \mathrm{e} / \AA^{3}$ intervals.

both the electron density of the clean Pd(111) surface and that of an isolated oxygen layer. The atomic positions of the Pd(111) and of the O-layer are taken to be the same as those of the relaxed adsorbate system. ${ }^{34}$ In this way, the presentation highlights the electronic structure and bond formation. Figure 7 depicts this difference electron density in a plane perpendicular to the surface. It is obvious that the perturbation created by the oxygen chemisorption is mostly localized on the oxygen atom itself and on its nearest neighbor $\mathrm{Pd}$ atoms. For $\mathrm{O}$ adsorbed in hcp sites we find essentially the same result.

As mentioned above, the difference electron density creates an electrostatic dipole moment. This dipole moment (besides a marginal contribution from the adsorbate-induced changes of the Pd surface geometry) equals the quantity $\mu$ displayed in Figure 6, and this is related to the adsorbate-induced work function change by the Helmholtz equation

$$
\mu=(12 \pi)^{-1} \mathrm{~A} \Delta \Phi / \theta
$$

Here the surface dipole moment is measured in Debye. The area of the $(1 \times 1)$ surface unit cell, $A$, must be given in $\AA^{2}$

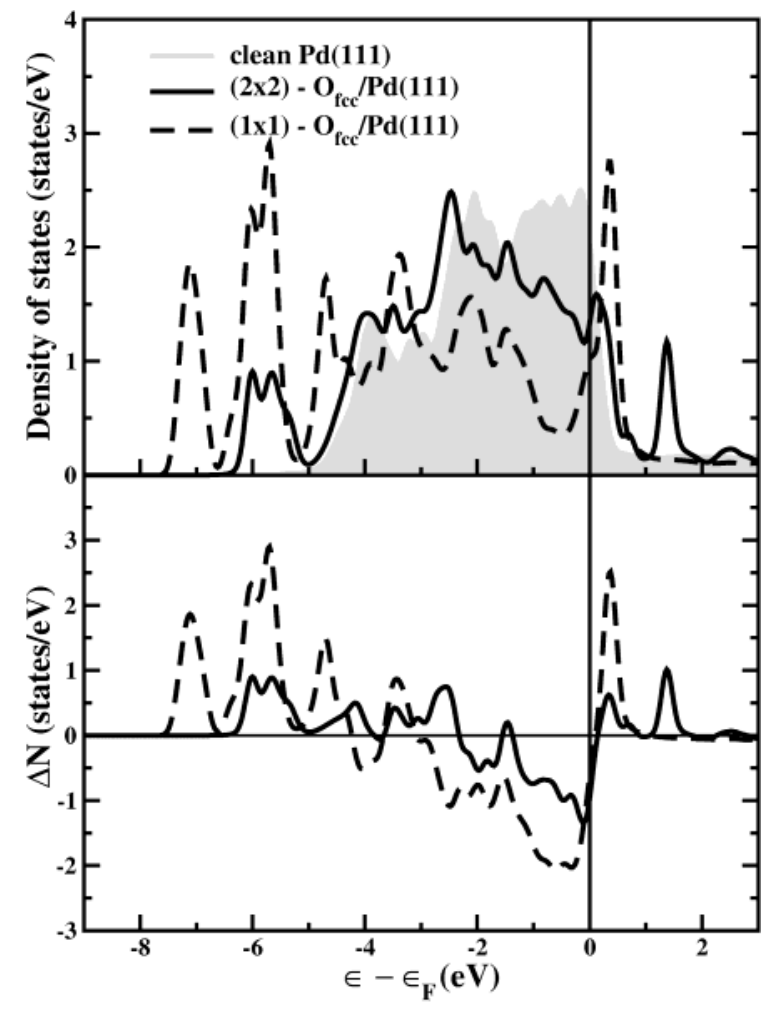

Figure 8. Top panel: Calculated local DOS for a 1-fold O-coordinated surface $\mathrm{Pd}$ atom in the $\mathrm{O}(2 \times 2) / \mathrm{Pd}(111)$ surface (solid line) and a 3 -fold $\mathrm{O}$-coordinated $\mathrm{Pd}$ atom in the $\mathrm{O}(1 \times 1) / \mathrm{Pd}(111)$ structure (dashed line). Also shown is the DOS of the top layer Pd atom of the clean surface as gray area. Bottom panel: corresponding differences of the DOS.

and the work function change $\Delta \Phi$ and coverage $\theta$ in $\mathrm{eV}$ and ML, respectively.

The adsorbate-adsorbate interaction, obviously, also affects the density of states (DOS). The DOS for the clean $\operatorname{Pd}(111)$ surface and the $\theta=0.25 \mathrm{ML}$ and $\theta=1 \mathrm{ML}$ overlayer structures (with $\mathrm{O}$ in fcc sites) are shown in Figure 8. In particular, the difference between the DOS of the clean and the adsorbatecovered surfaces clearly reveal the $\mathrm{O}-\mathrm{Pd}$ bonding and antibonding states. They are just below and just above the nearly fully occupied palladium d band: bonding states around 5-8 $\mathrm{eV}$ below the Fermi level and antibonding states around and just above $\epsilon_{\mathrm{F}}$.

For the full monolayer case, we see 4 peaks at the lower edge of the $d$ band: one peak at $-7.2 \mathrm{eV}$, a double peak around $-5.8 \mathrm{eV}$, and peak at $-4.7 \mathrm{eV}$. Obviously, at this coverage we expect the formation of an adsorbate bandstructure,,$^{35}$ and this is shown in Figure 8. The three lower DOS peaks ( -7.2 and $-5.8 \mathrm{eV}$ ) are clearly due to the oxygen p orbitals (more precisely due to the bonding states the $\mathrm{O} 2 \mathrm{p}$ orbitals form with the Pd $\mathrm{d}$ band). The $-4.7-\mathrm{eV}$ peak has mainly d character. It is due to a state that at the $\bar{K}$ point splits off the bulk d band. The presence of a noticeable oxygen-oxygen interaction in the overlayer is clearly reflected, for example, in the $2-\mathrm{eV}$ bandwidth of the bandstructure of the bonding states. The antibonding $\mathrm{O}-$ metal states are also visible in the bandstructure plot, namely, around $-2 \mathrm{eV}$ and just above the Fermi level.

It is instructive to compare these findings with the results available for $\mathrm{O}$ on the other late $4 \mathrm{~d}$ TMs. ${ }^{14-16}$ Concerning the DOS the obvious difference between $\mathrm{Ru}, \mathrm{Rh}, \mathrm{Pd}$, and $\mathrm{Ag}$ is the progressively lower-lying $\mathrm{d}$ band with respect to the Fermi level, yielding a half-filled band for Ru and a fully occupied band for Ag. Since in all these systems the O-metal interaction gives 


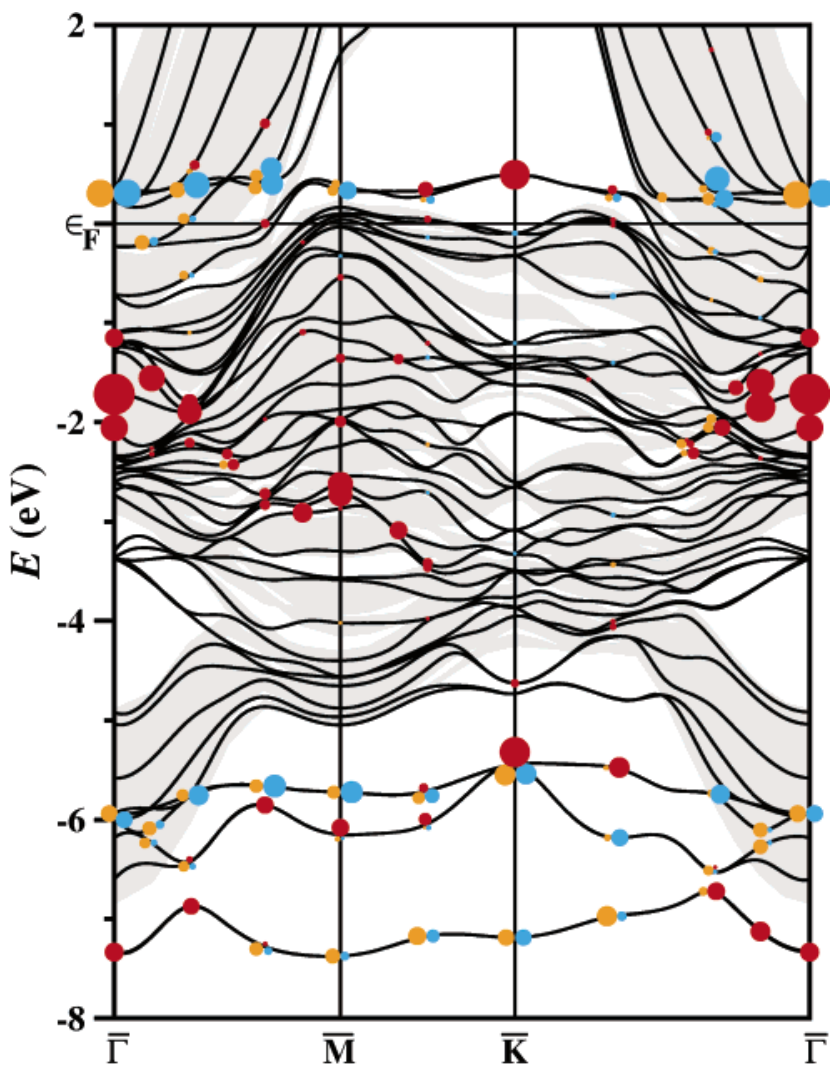

Figure 9. Surface band structure of $\mathrm{O}(1 \times 1) / \mathrm{Pd}(111)$ (solid lines). The gray area corresponds to the projected Pd bulk band structure, and the circles along the lines reflect the oxygen $\mathrm{p}$ orbital character of the bands: $\mathrm{p}_{x}$ (orange); $\mathrm{p}_{y}$ (blue); $\mathrm{p}_{z}$ (red). (The radius of the circles scales with the projection onto the corresponding spherical harmonics within the oxygen muffin tin sphere.)

rise to bonding states at the lower edge of the $\mathrm{d}$ band and antibonding states at the upper edge of the $d$ band, the antibonding states become progressively more filled toward the right of the periodic table, thus reducing the bond strength. With decreasing importance of $\mathrm{d}$ bonding, the O-induced outward relaxation of the first layer is smaller for $\mathrm{Rh}$ than for $\mathrm{Ru}$, and for $\mathrm{Ag}$, the then-dominant reduced sp pressure leads to a slight contraction, cf. section IIIA. We speculate that the trend reversal in $\mathrm{Pd}$ at high $\mathrm{O}$ coverages, i.e., the unusual contraction in the $\mathrm{O}(1 \times 1)$ structure, is a consequence of the transition from one regime to the other. Corresponding changes in the electronic structure leading to this crossover are very subtle and thus difficult if not impossible to identify. Related to this physics and competition of mechanisms is our finding that the potential energy surface $E\left(d_{12}\right)$ at higher $\mathrm{O}$ coverages is extremely flat, i.e., at finite temperatures the system will undergo large fluctuations. Molecular dynamics studies of this system are currently being performed. We also note that even despite the comparably appreciable difference in the binding energies for $\mathrm{O}$ at the late $4 \mathrm{~d}$ TM series, for example, the difference electron density for $\mathrm{O} / \mathrm{Ru}, \mathrm{O} / \mathrm{Rh}, \mathrm{O} / \mathrm{Pd}$, and $\mathrm{O} / \mathrm{Ag}$ still look very similar, as may be verified by comparing the published plots for the $\mathrm{O}(2 \times 2)$ phase on $\mathrm{Ru}(0001),{ }^{14} \mathrm{Rh}(111),{ }^{15} \mathrm{Pd}(111)$, cf. Figure 7, and $\operatorname{Ag}(111){ }^{16}$

For the work function, the adsorption-induced increase as function of the oxygen coverage is similar for $\operatorname{Pd}(111)$ $\left(\Delta \Phi_{\mathrm{O} / \mathrm{Pd}(111)}(\theta=1 \mathrm{ML})=1.54 \mathrm{eV}\right), \operatorname{Ru}(0001)\left(\Delta \Phi_{\mathrm{O} / \mathrm{Ru}(0001)}(\theta\right.$ $=1 \mathrm{ML})=1.30 \mathrm{eV})$, and $\operatorname{Rh}(111)\left(\Delta \Phi_{\mathrm{O} / \mathrm{Rh}(111)}(\theta=1 \mathrm{ML})=\right.$ $1.23 \mathrm{eV})$. For $\operatorname{Ag}(111)$, on the other hand, the change is more than twice as large: $\Delta \Phi_{\mathrm{O} / \mathrm{Ag}(111)}(\theta=1 \mathrm{ML})=3.73 \mathrm{eV} \cdot{ }^{14-16}$
Furthermore, for the $\mathrm{Ru}$ and $\mathrm{Rh}$ substrates, $\Delta \Phi(\theta)$ increases nearly linearly up to $\theta=0.75 \mathrm{ML}$, and then a saturation behavior is found, i.e., for $0.75<\theta \leq 1 \mathrm{ML}$, the work function remains approximately constant. ${ }^{14,15}$ As apparent from Figure $6, \operatorname{Pd}(111)$ does not show such a saturation behavior, and neither does $\operatorname{Ag}(111) .{ }^{16}$

\section{Conclusions}

In this paper, we presented a systematic DFT-GGA study of the on-surface adsorption of oxygen at $\operatorname{Pd}(111)$ in the submonolayer coverage range. In particular, we provide data on energies, geometries, and the electronic structure. Comparison with earlier studies on $\mathrm{O} / \mathrm{Ru}(0001), \mathrm{O} / \mathrm{Rh}(111)$, and $\mathrm{O} / \mathrm{Ag}(111)$ enables us to discern some trends in the oxygen bonding at these late $4 \mathrm{~d}$ transition metal surfaces. The continued filling of the metal $\mathrm{d}$ band yields a progressive weakening of the bond strength that in turn has consequences on the surface relaxations. Within this TM series Pd represent an interesting borderline case: At low $\mathrm{O}$ coverages $\mathrm{Pd}(111)$ shows some similarities with $\mathrm{Ru}(0001)$ and $\mathrm{Rh}(111)$, and at $\theta=1 \mathrm{ML}$ the relaxation characteristics is more similar to $\mathrm{O} / \mathrm{Ag}(111)$. Still, there are no pronounced indications of a qualitative change in the nature of the $\mathrm{O}-\mathrm{Pd}$ on-surface chemical bond at low and high oxygen on-surface coverage. As already discussed before, ${ }^{1}$ we attribute the propensity of $\mathrm{Pd}(111)$ to form (surface) oxides for $\mathrm{O}$ coverages with $\theta>0.5 \mathrm{ML}$ rather to the relative stability of subsurface oxygen, the details of which will be the focus of a subsequent publication. ${ }^{35}$

Acknowledgment. We gratefully acknowledge discussions with Veronica Ganduglia-Pirovano, Weixue Li, and Cathy Stampfl. We also thank Cathy Stampfl for critically reading the manuscript. This work was partially supported by the Deutsche Forschungsgemeinschaft (Schwerpunkt "Katalyse").

\section{References and Notes}

(1) Todorova, M.; Li, W. X.; Ganduglia-Pirovano, M. V.; Stampfl, C.; Reuter, K.; Scheffler, M. Phys. Rev. Lett. 2002, 89, 096103.

(2) Reuter, K.; Stampfl, C.; Ganduglia-Pirovano, M. V.; Scheffler, M. Chem. Phys. Lett. 2002, 352, 311.

(3) Li, W. X.; Stampfl, C.; Scheffler, M. Phys. Rev. B 2003, 67, 045408.

(4) Li, W. X.; Stampfl, C.; Scheffler, M. Phys. Rev. B 2003, 68, 165412.

(5) (a) Ertl, G. J. Mol. Catal. A 2002, 182-183, 5. (b) Engel, T.; Ertl, G. Oxidation of Carbon Monoxide. In The Chemical Physics of Solid Surfaces and Heterogeneous Catalysis; King, D. A., Woodruff, D. P., Eds.; Elsevier: Amsterdam, 1982. 245.

(6) Conrad, H.; Ertl, G.; Küppers, J.; Latta, E. E. Surf. Sci. 1977, 65,

(7) Légaré, P.; Hilaire, L.; Maire, G.; Krill, G.; Amamou, A. Surf. Sci. 1981, 107, 533.

(8) Imbihl, R.; Demuth, J. E. Surf. Sci. 1986, 173, 395 5787.

(9) Guo, X.; Hoffman, A.; Yates, J. T., Jr. J. Chem. Phys. 1989, 90 ,

(10) Banse, B. A.; Koel, B. E. Surf. Sci. 1990, 232, 275

(11) Voogt, E. H.; Mens, A. J. M.; Gijzeman, O. L. J.; Geus, J. W. Surf. Sci. 1997, 373, 210.

(12) Seitsonen, A. P.; Kim, Y. D.; Schwegmann, S.; Over, H. Surf. Sci. 2000, 468, 176.

(13) Lundgren, E.; Kresse, G.; Klein, C.; Borg, M.; Andersen, J. N.; De Santis, M.; Gauthier, Y.; Konvicka, C.; Schmid, M.; Varga, P. Phys. Rev. Lett. 2002, 88, 246103.

(14) Stampfl, C.; Scheffler, M. Phys. Rev. B 1996, 54, 2868.

(15) Ganduglia-Pirovano, M. V.; Scheffler, M. Phys. Rev. B 1999, 59, 15533.

(16) Li, W. X.; Stampfl, C.; Scheffler, M. Phys. Rev. B 2002, 65, 075407.

(17) Blaha, P.; Schwarz, K.; Luitz, J. WIEN97, A Full Potential Linearized Augmented Plane Wave Package for Calculating Crystal Properties; Technical Universität Wien: Austria, 1999; ISBN 3-9501031$0-4$.

(18) Kohler, B.; Wilke, S.; Scheffler, M.; Kouba, R.; Ambrosch-Draxl, C. Comput. Phys. Commun. 1996, 94, 31. 
(19) Petersen, M.; Wagner, F.; Hufnagel, L.; Scheffler, M.; Blaha, P.; Schwarz, K. Comput. Phys. Commun. 2000, 126, 294.

(20) Perdew, J. P.; Burke, K.; Ernzerhof, M. Phys. Rev. Lett. 1996, 77, 3865 .

(21) Reuter, K.; Ganduglia-Pirovano, M. V.; Stampfl, C.; Scheffler, M. Phys. Rev. B 2002, 65, 165403.

(22) Kittel, C. Introduction to Solid State Physics, 6th ed.; Wiley: NY, 1986.

(23) Honkala, K.; Laasonen, K. J. Chem. Phys. 2001, 115, 2297.

(24) Ohtani, H.; Van Hove, M. A.; Somorjai, G. A. Surf. Sci. 1997, 187,372 .

(25) Felter, T. E.; Sowa, E. C.; Van Hove, M. A. Phys. Rev. B 1989, 40,891 .

(26) Barbieri, A.; Van Hove, M. A.; Somorjai, G. A. Surf. Sci. 1994, 306,261 .

(27) Maca, F.; Scheffler, M.; Berndt, W. Surf. Sci. 1985, 160, 467

(28) Christmann, K.; Ertl, G.; Schober, O. Surf. Sci. 1973, 40, 61.
(29) $\mathrm{Ru}(0001)$ data: Reuter, K., unpublished. Basis set as described in Phys. Rev. B 2002, 65, 165403. Ag(111) FP-LAPW basis set: $R_{\mathrm{MT}}^{\mathrm{Ag}}=2.36$ bohr, $R_{\mathrm{MT}}^{\mathrm{O}}=1.30 \mathrm{bohr}, l_{\max }^{\mathrm{wf}}=12, l_{\max }^{\mathrm{pot}}=4$, and local orbitals for the $4 \mathrm{~s}$ and $4 \mathrm{p}$ semicore states of $\mathrm{Ag}$, as well as for the O $2 \mathrm{~s} . E_{\max }^{\mathrm{wf}}=17 \mathrm{Ry}, E_{\max }^{\mathrm{pot}}$ $=169 \mathrm{Ry},(12 \times 12 \times 01) \mathrm{MP}$ grid

(30) Methfessel, M.; Hennig, D.; Scheffler, M. Phys. Rev. B 1992, 46, 4816 .

(31) Steltenpohl, A.; Memmel, N. Surf. Sci. 1999, 443, 13.

(32) Stampfl, C.; Kreuzer, H. J.; Payne, S. H.; Pfnür, H.; Scheffler, M. Phys. Rev. Lett. 1999, 83, 2993.

(33) Demuth, J. E. Chem. Phys. Lett. 1977, 45, 12.

(34) Scheffler, M.; Stampfl, C. Theory of Adsorption on Metal Substrates. In Handbook of Surface Science, Vol. 2: Electronic Structure; Horn, K., Scheffler, M., Eds.; Elsevier: Amsterdam, 2000.

(35) Todorova, M.; Reuter, K.; Scheffler, M., in preparation. 